\title{
Hacia el nuevo Hospital Central de la Defensa «Gómez Ulla"
}

Durante los últimos años los Centros Sanitarios, están experimentando una serie de cambios motivados por la propia evolución de la sociedad. La sanidad ocupa un lugar prioritario en nuestro entorno socioeconómico y en las últimas décadas ha aumentado la sensibilidad social respecto a los servicios sanitarios y sus efectos, aumentando el nivel de exigencia de sus usuarios.

En España, la sanidad es una de las competencias transferidas por el Gobierno Central, que más peso específico mantiene en los presupuestos de las Comunidades Autónomas que han asumido dicha gestión, como la Comunidad de Madrid, lo que conlleva de forma continua a establecer nuevas formas de gestión, para aumentar su eficiencia.

Todos estos cambios afectan a la Sanidad Militar, y a sus Centros Sanitarios, como centros de referencia de entrenamiento de sus profesionales médicos y enfermeros. De ahí la importancia de la modernización a nivel administrativo, técnico y humano, de los centros sanitarios militares y no retrasarse con respecto a otros centros sanitarios, de su mismo nivel, y con ello estar capacitados para cumplir su función asistencial en territorio nacional y en las proyecciones sanitarias militares en el extranjero.

En el año 2007, se aprobó el Plan Director del Hospital Central de la Defensa, que esta permitiendo la actualización estructural del edifico y de distintos departamentos como: centralización de consultas externas en la planta baja y primera, unificación de laboratorios, bloque quirúrgico, servicio de urgencias, unificación del Área Docente, etc. Este plan también ha dado lugar a la adquisición de aparatos de electromedicina de última generación y al establecimiento de un nuevo Sistema de Información Sanitaria, con capacidad para la integración de forma completa de toda la documentación clínica generada en la asistencia sanitaria de un paciente, así como las pruebas diagnósticas y procedimientos terapéuticos que se generen en esta asistencia.

Los items descritos anteriormente, junto con el desarrollo de una plantilla de profesionales ajustada a un entorno poblacional, han permitido que el Hospital Central de la Defensa, sea un Centro Sanitario capacitado, para abrirse a la población cercana geográficamente y a la que quiera elegir este Centro
Sanitario como el adecuado para tratar su enfermedad. Con ello culmina el Convenio de Colaboración, entre el Ministerio de Defensa y la Comunidad de Madrid en materia de asistencia sanitaria.

El Hospital Central de la Defensa, establece como línea de trabajo, los objetivos característicos de la nueva idea de hospitales, como propios. Entre estos objetivos cabe destacar:

Mejoría de la seguridad del paciente.

Mejoría del acceso a la asistencia especializada.

Aumento de la eficiencia.

Alcanzar la equidad para los pacientes.

Prevención de la enfermedad.

Para poder ir cumpliendo estos objetivos, el Hospital Central de la Defensa, al igual que otros hospitales de la Comunidad de Madrid, se tendrá que afianzar en la llamada Reconfiguración hospitalaria, que tiene como finalidad la mejora de la salud, calidad asistencial y la asunción de continuidad del proceso.

En el proceso de Reconfiguración hospitalaria, se necesitan establecer cambios, algunos de ellos ya se están realizando en el Hospital Central de la Defensa como: el aumento de los procesos de ambulatorización, la disminución de la estancia media por proceso asistencial, la disminución del número de camas. Otros cambios van a ser inmediatos como: la apertura de los profesionales sanitarios a la Libre Elección. Y otros cambios se irán estableciendo en el futuro como: la organización de servicios especiales, reagrupación estructural en servicios donde exista el mayor entrenamiento posible por tener un gran número de casos y con ello aumentar el entrenamiento de los profesionales y la supervivencia de los pacientes.

En este periodo de cambio, se están estableciendo los pilares, que sostendrán el futuro del Hospital Central de la Defensa, como centro sanitario militar y como centro de referencia para la población madrileña.

Ángel Fernández Díez

Director Gerente del Hospital Central de la Defensa «Gómez Ulla» 